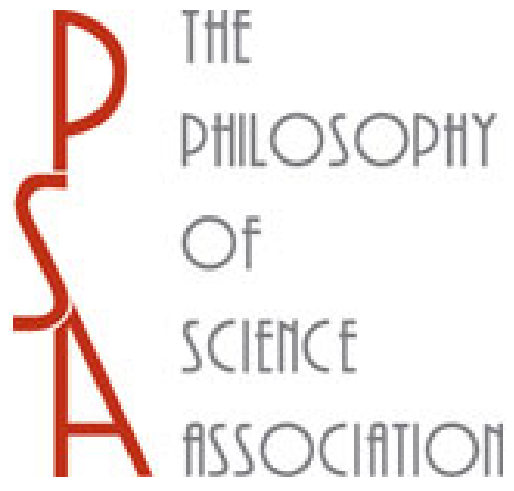

Critical Points in Modern Physical Theory

Author(s): Henry Margenau

Source: Philosophy of Science, Vol. 4, No. 3 (Jul., 1937), pp. 337-370

Published by: The University of Chicago Press on behalf of the Philosophy of Science Association

Stable URL: http://www.jstor.org/stable/184447

Accessed: 18/07/2011 17:11

Your use of the JSTOR archive indicates your acceptance of JSTOR's Terms and Conditions of Use, available at http://www.jstor.org/page/info/about/policies/terms.jsp. JSTOR's Terms and Conditions of Use provides, in part, that unless you have obtained prior permission, you may not download an entire issue of a journal or multiple copies of articles, and you may use content in the JSTOR archive only for your personal, non-commercial use.

Please contact the publisher regarding any further use of this work. Publisher contact information may be obtained at http://www.jstor.org/action/showPublisher?publisherCode=ucpress.

Each copy of any part of a JSTOR transmission must contain the same copyright notice that appears on the screen or printed page of such transmission.

JSTOR is a not-for-profit service that helps scholars, researchers, and students discover, use, and build upon a wide range of content in a trusted digital archive. We use information technology and tools to increase productivity and facilitate new forms of scholarship. For more information about JSTOR, please contact support@jstor.org. 


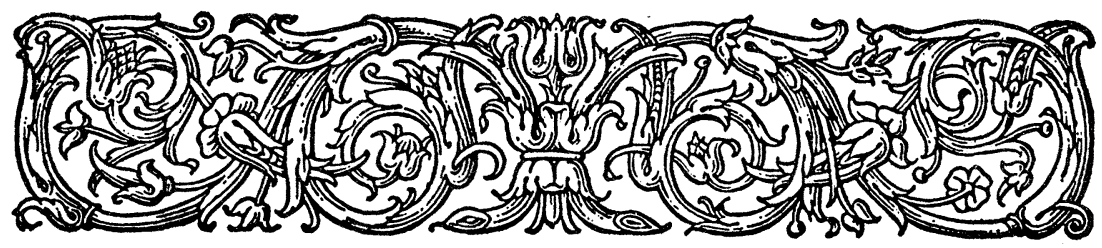

\section{Critical Points in Modern Physical Theory}

BY

\section{HENRY MARGENAU}

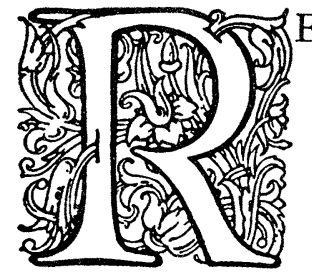

ECENT discussions in the physical literature, ${ }^{1}$ designed to clarify the logical position of modern physical theory, have brought to light an amazing divergence of fundamental attitudes which may well bewilder the careful student of physics as well as philosophy. Quantum mechanics, representing an abstract formalism, should be capable of having its logical structure analyzed with great precision like any other mathematical discipline. Its consequences in all problems to which its method can be applied are so unambiguous, consistent, and successful in predicting physical experience as to disperse immediately all thoughts of possible discrepancies in its fundamental texture. Yet it must be said that even the founders of quantum theory are not in harmony in their various expositions of the bases of that theory. However, while this situation seems disquieting on the face of it, there is no cause for serious brow raising, for it is a fact that there exists agreement with regard to the central axioms of the theory, and that the ambiguities affect only their philosophical interpretation, a field in which differences of opinion may at present be honestly entertained.

In the present article we desire to draw attention to several ways in which these axioms can be and have been interpreted. Without attempting finality we shall investigate the consequences

${ }^{1}$ See bibliography at the end of this paper. 


\section{Critical Points in Physical Theory}

of different views and separate clearly their incompatible features, appraising now and then the various possibilities as to their reasonableness and their degrees of popularity among scientists. In the course of this endeavor there will arise occasional suggestions dealing with wide-spread terminology, whose aim will be to guard against confusion of language and to eliminate certain annoying contradictions the origin of which is purely verbal. There will be an analysis and proposed definitions of such important terms as state and measurement, for it is believed that they have not yet acquired universal meaning and are for that reason the chief source of confusion in discourses on the foundation of modern physics. We begin by outlining those axioms of the new quantum mechanics on which the description of nature essentially depends.

\section{THE AXIOMS OF QUANTUM MECHANICS ${ }^{2}$}

There are two ways in which the basic postulates of any theory may be presented: the inductive and the deductive. Textbooks on quantum mechanics generally use the former. They start by giving an account of the failures of classical physical description, then proceed to involve the reader in the intracacies of measuring devices, tell him with enormous emphasis that there is absolutely no hope of ever performing a measurement which achieves simultaneous accuracy for complementary (conjugate) physical quantities, dazzle him with Heisenberg's uncertainty relations and finally introduce him to the use of matrices and general operators. This process is awe-inspiring and pedagogically effective with minds that wish to learn rapidly. But it leaves unsatisfied the student who is interested in fundamental questions of meaning, for the steps lack logical compulsion and uniqueness. In the first place, no inductive proof, based on experiments, can ever be given for the impossibility of increasing the accuracy with which canonically conjugate observables can be measured. Secondly, the complete meaning of indeterminacy is difficult to convey by examining individual measurements alone,

${ }^{2}$ Only a general discussion is here intended. For a complete statement see P. A. M. Dirac, Principles of Quantum Mechanics, or Lindsay and Margenau, Foundations of Physics, p. 40 r. 
and finally, the particular choice of operators which is made in quantum mechanics does not follow uniquely from the uncertainty relations. It may be that the general use which this particular mode of presentation enjoys everywhere is partly responsible for the uncritical attitude toward basic matters which many working physicists have so long displayed.

The second, the deductive method, does not pretend to prove anything new - a semblance which often attends the former procedure-but resigns itself to an enumeration of a new set of axioms in terms of which we agree tentatively to describe our physical experience. The analysis of the situation thus created, once the postulates are stated, is a matter of complete logical and mathematical rigor, and the consequences are capable of experimental test. Success in fitting data strengthens the belief that the initial axioms are a suitable starting point in physical investigations, though it cannot convey the conviction that these axioms are unique, for it might well be that another set were equally successful. This question of uniqueness will probably never be settled, and it is a fortunate circumstance that we have no means for doing so, a circumstance which prevents our theories from freezing into rigid patterns, which keeps our inspiration and our hopes alive. But strongest confidence in the essential "correctness" of the axioms, which have proven their worth in empirical predictions, comes when we begin to interpret them and find that they differ from the postulates of classical physics precisely at those points where critical analysis has already shown the latter to be defective. In this manner the new theory acquires a degree of certainty which is the maximum attainable. This form of presentation, which exposes more truly the logical status of quantum mechanics and provides a ready answer to the question of its ultimate correctness, will here be chosen although it does not possess the obvious pedagogical advantages of the former method.

Physical analysis deals with the behavior, imagined or observed but always in principle observable, of physical systems. ${ }^{3}$ Quantum

3 Physical systems are in general constructs in the sense of a previous communication (Journal of Phil. of Science, 2, 48 (I935); 2, I64 (I935)). We shall here assume the terms to be so understood. 


\section{Critical Points in Physical Theory}

mechanics, in particular, has to do with systems observable only by indirect means, principally because of their smallness. To analyze their behavior the physicist invents states. Now in common language a state carries with it the connotation of some mysterious objective reality, whatever that word may mean; it implies something entirely independent of our description, something to which our description must conform. As a matter of physical convention, however, a state is deprived of this exalted independence and is entirely at our mercy. We determine how we are going to describe states, and the agreement with respect to this matter constitutes the first axiom of any theory. In classical mechanics states were defined, or specified, by means of coordinates and momenta of mass points; in thermodynamics an altered convention is being used: by state is meant a coordination of two thermodynamic variables, such as pressure and volume of a system. In quantum mechanics, finally, we agree to mean by state, or the representative of a state, a certain function of a suitable number of variables.

The distinction between state and representative of a state has here been made merely as a concession to ultra-meticulous readers. Such a difference exists only on the scale of ordinary experiences where an intuitive conception of the coordinates and momenta of a body, as a bullet in flight, is obtainable. Here one can associate an unambiguous mental picture with the state which has as its representative a certain group of positions and momenta. On the atomic scale, where no such picture can be obtained except by intrinsically unverifiable extrapolation of large scale experience, the dangers of which are now quite apparent, it seems in order to drop the distinction and refer to the "representative of a state," which alone is available, briefly as the state.

But the introduction of a state function is exceedingly abstract and is not at all linked with physical experience. What is the operational meaning of this procedure? This leads to an important point. It is true that classical physics did formulate its states in terms of measurable quantities, as well it might, for it operated on a scale of visual perception. But this should be regarded as a coincidence rather than a logical necessity: there is 
no consideration which compels that states be directly measurable. All properties, to be sure, with which physical systems are endowed, must be measurable. States are invented in order to enable statements regarding measurable physical properties to be made, which is by no means synonymous with saying that states must be measurable. In quantum mechanics it happens indeed that states become intermediators between abstract constructs called systems and their measurable properties.

The invention of states which, while they are themselves not observable, would also fail to provide a definite connection with experience, must be considered as futile. But quantum mechanics is not in such sterile condition. By means of a second axiom a very precise link is made between a system characterized by a given state and its observable properties. The connection is not of the usual reversible type but involves probability aggregates. The axiom posits that when a state function is given, the exact probability distribution with regard to every observable quantity can be computed. The detailed manner in which this is to be done is not relevant to the main theme of this discussion. We have thus established a one-one correspondence, not between states and observed properties, but between states and probability distributions of observed properties. If causality were to be defined as residing in a unique transition between state and observation, then the description to which this axiom gives rise would have to be termed non-causal. Practically speaking, when the state of a system is known, the probability that a given value of the momentum shall be observed can be predicted, but the outcome of a given momentum measurement is in general obscure. One of the notable consequences of this axiom is Heisenberg's famous principle of related indeterminacies. It states that the standard deviation of the probability distribution with respect to the momentum of a system multiplied by the standard deviation of the probability distribution with respect to its measured position is never smaller than a certain universal constant (Planck's $h$ divided by $4 \pi$; momentum and position may here, of course, be replaced by any other two so-called canonically conjugate quantities). The reader who is unfamiliar with the statis- 


\section{Critical Points in Physical Theory}

tical term "standard deviation" may in this connection think qualitatively of the spread of the measurements.

We have already indicated that on the basis of the present formalism a single measurement can never suffice to determine a state. This leads at once to the important question: Can the state function be inferred at all, even from a number of observations sufficient to determine all probabilities with respect to a given observable (measurable quantity)? The answer is not an unqualified yes. Let us take a simple example. We wish to determine by observation the state function of an electron about which nothing is known at present. For this purpose we might decide to measure its energy. According to our preceding considerations, we must not expect to find the same energy value every time the measurement is repeated on the system in the same state (supposing here that we have means of reproducing the state; cf. below). Hence we are forced to determine how many measurements yield the value $E_{1}$, how many yield the value $E_{2}$ and so forth. If we perform altogether I000 measurements, IOO of which have given the value $E_{1}$, we say that the probability of that occurrence is I/Io etc. Finally we shall be in possession of a sequence of numbers $\mathrm{p}_{1}, \mathrm{p}_{2}, \ldots$, the probabilities of the individual measured values, the totality of which constitutes the probability distribution. The point is that from this knowledge the state function can not be constructed. It involves, not the p-values, but numbers characterized merely by the requirement that the squares of their absolute values shall equal the p's, and this requirement is not sufficient to specify these numbers completely. Thus it would be impossible in our example to predict the probable distribution of momentum measurements on the basis of the results obtained with regard to the energy. ${ }^{4}$ This proposition is proved in detail in the appendix (AI).

The situation here described is indeed disturbing; it would seem to require that the description of states adopted in quantum mechanics is forever aloof from physical experience, that states can never be known completely through observation. But this

${ }^{4}$ Excluding, of course, those special cases, like the free electron, where energy and momentum operators commute. 
is not the case, at least not in principle. Suppose, for instance, that our energy measurements had all yielded the same value, which is entirely possible. Then we should have known the state function with sufficient completeness to predict the probability distribution with respect to every other type of measurement. It is true in general that if a given kind of observation does not spread, the statistical distribution governing the observations on all other quantities can be inferred, and this is the only condition under which such an inference is possible. We are now in possession of the clue which can ideally lead to an experimental determination of any state function: If the particular kind of observation which we happen to perform is seen after a few trials to spread, we proceed to measure other quantities until one is found with respect to which the state is "pure," i.e., which yields the same value in all trials.

But now we come to the troublesome question: does such a quantity exist? Nobody knows. If this answer implies, in good positivistic fashion, that such a quantity does not exist, then the matter is settled for the time being: a state function can not in general be found, or constructed, on the basis of measurements. Such a position, by the way, is entirely tenable and does not reflect in the least upon the usefulness or legitimacy of quantum mechanics as a theory. But let us not dispense with this matter too hastily. There are, after all, those who hold that the number $\pi$ existed before it was discovered.

The question as to the existence of a quantity whose measurements will not spread for an arbitrary state function resolves itself on closer analysis in to two; first, the mathematical one: does an arbitrary function of a fixed number of variables, which satisfies certain (boundary) conditions, possess an operator with respect to which it is an eigenfunction? second, is it possible to associate with every operator an operation which could be regarded as a measurement of a physical quantity? It is clear that the number of possible mathematical operators as well as the number of physical operations is infinite, so that there is an unlimited choice. While it is at present a pious hope that the difficult program of correlating functions, operators, and physical 


\section{Critical Points in Physical Theory}

operations in the unique manner here required will ever be carried out, there is at least no basic obstacle in the way of its execution. The fact is of course that the interest of physicists has been limited to relatively few physical quantities which can be represented by about half a dozen different operators, and that research has been remarkably successful despite this limitation. On the whole it seems not unreasonable to answer the question at the beginning of this paragraph in the affirmative, and thereby to provide an ideal basis for linking states with observations.

The first axiom had to do with the definition of states; the second dealt with the relation between states and experience and involved several matters which can easily give rise to misunderstanding. We now take up the thread of the original discourse and introduce the third and final axiom. It is clear and concise and requires very little comment. It amounts to a recipe for determining what possible values any given physical quantity can assume when a measurement is made. In barring much, it imposes a most daring restriction upon experience and exposes itself to exceedingly severe tests. Suffice it to say that it has successfully survived many tests and may be said to be in good standing. The structure of this axiom is mathematical; it states that only eigenvalues of operators associated with physical observables can appear as the results of measurements. But a complete technical understanding of these matters is hardly necessary for the subsequent discussion.

\section{CLOSER ANALYSIS OF THE MEANING OF STATES}

We now leave aside the factual content of the postulates and propose to view them-as far as we are able to do so-from the vantage point of the philosopher. At the same time we are entering upon controversial ground. States were said to be (represented by) certain functions having an indirect but definitely specifiable connection with possible experiences. They served to describe the behavior of physical systems. Let us be more precise and examine whether these states describe physical systems or merely our knowledge of physical systems. The answers to several controversial questions depend on the stand which is 
taken on this particular issue as will appear later, and it is very surprising that the importance of a clear-cut decision in this matter has rarely been recognized even by the most authoritative writers. It is difficult to find an article in which the author, though evidently speaking from one point of view, does not somewhere permit himself at least a verbal lapse toward the other. A middle stand (or shall we say seat, for this is by far the most comfortable position) is taken by many who pronounce the question as meaningless and consequently recognize no distinction. This possibility must also be investigated.

The contrast between physical systems and our knowledge of physical systems is not as deep as the epistomological difference between realism and subjective idealism, and the philosopher who hopes for a decision between these issues from any physical theory will be disappointed. No physical theory is tied to any such metaphysical basis. Classical physics made sense to Newton as it did to Berkeley, the only difference being that Newton thought of physical systems as objective entities transcending consciousness, while Berkeley regarded them as existing within consciousness, but nevertheless "existing." So in quantum mechanics: whether we place physical systems outside ourselves and contrast them with conscious knowledge of them in a fundamental epistemological sense, or whether we recognize, within consciousness, a difference between knowable systems and knowledge of them, the systems themselves being merely constructs-the choice does not matter as long as we maintain an intelligible distinction between the concept of system and that of knowledge about the system. This distinction, it seems, is so thoroughly ingrained in our thinking, so vividly alive in our intuition that it would be difficult to eradicate even if there were logical or metaphysical grounds for doing so. Or must we, after all, yield to superior logic and admit that the distinction fails? If the electron has no transcendental "reality" but is only a construct with which we operate, does it not, as a concept, become identical with our knowledge, i.e., awareness of it? It seems that, unless we are willing also to confuse the existence of the solution of an equation with our knowledge of that solution, the answer must be no. But 


\section{Critical Points in Physical Theory}

there are in many cases definite rules for telling whether a solution exists, while to know the solution would be an altogether different matter. Logically, we feel that the distinction must be maintained, and this is fortunate, for otherwise logic would painfully contradict our in tuition.

Having cleared away the metaphysical underbrush we may now proceed with greater ease. Does the quantum mechanical state function refer to the system, or does it describe our knowledge? Planck, who according to his recent writings professes a very wholesome realism, would seem to favor the first alternative. The spirit of Dirac's writings, although they contain no specific pronouncements, indicates that his states refer to systems apart from our awareness. Schrödinger, on the other hand, speaks occasionally of the state function as the "maximal sum of knowledge," indicating perhaps a preference for the second view. Bohr and Heisenberg mention frequently the conflict between observer and observed object with their unavoidable interaction and thereby presumably express themselves in favor of an objective world, the properties of which are to be described according to the first view. But this last diagnosis may be in error.

Let it be said at once that neither position can be wrong in the sense that it leads to erroneous consequences if carried through correctly. We mean here consequences of a positively verifiable character. The logical implications are in some respects very different, and this is particularly true about the meaning of probability in the two cases. But before examining the necessities of interpretation enforced by each of the two points of view, it may be well to pause for a moment and to see if one of them is not more plausible, or more in line with other accepted doctrines than the other.

In common language, which after the foregoing orienting excursions may perhaps be used without fear of misinterpretation, the distinction in question is simply that between physical objects and the observer's knowledge about physical objects. In classical mechanics the distinction was extremely sharp, and it was supposed that the two, besides being generically different as logical entities, were independent even in a physical sense. States 


\section{H. Margenau}

clearly referred to the objects themselves, positions and momenta were their direct properties. The objectivity of states had its culminating expression in the equations of motion, which were understood to imply actual space-time propagation of systems. This propagation took place according to rigid laws independently of the observer's knowledge.

Such an extreme view regarding the independent objectivity of physical states cannot be carried over into quantum mechanics; there must at least be a shift of emphasis. One of the great discoveries at the beginning of the present era in physics was the recognition that objects and knowledge are related, because the classical notion of ideally unlimited accuracy of experimental devices failed and made classical knowledge intrinsically impossible. Hence if the view of objectivity be adopted, its classical meaning must be modified to this extent. It goes without saying that states must also be stripped of all impediments pertaining to sensual perception, and thought of entirely in abstracto. Psychological objections to this procedure, frequently raised by members of the older school, are of no particular moment in this connection, for it is not a matter of convenience, but one of logic which concerns us here.

The objective view of quantum mechanical states is enormously strengthened by the fact that states develop in time according to a definite differential equation (Schrödinger's) which has a form not unlike the equations of motion in classical physics. It is true that the simple interpretation of spatio-temporal propagation presents its difficulties or is at any rate no longer in tuitively direct, but the fact remains that there are determinate changes in time which are difficult to correlate with simultaneous changes in the observer's knowledge because they seem enforced dynamically and not psychologically.

There are further points which may be cited as evidence for the plausibility of the objective view. We have seen (third axiom) that quantum mechanics makes very drastic positive predictions about possible experience, predictions which do not resemble laws of thought and give an impression of utter independence of human knowledge. To be sure this axiom has little to do with the 


\section{Critical Points in Physical Theory}

definition of states, but it does indicate that part at least of the new formalism concerns itself with matters that are thoroughly factual in a sense distinct from mere knowledge. And if this is true there seems to be little motive for insisting that another concept, that of state, should deal not with factual objects but with our modes of awareness.

It was pointed out that classical mechanics in all its structure suggests the objective view. Now it is possible to show that, if applied to large scale bodies, quantum mechanics leads to the same physical consequences as does the classical theory. The latter may be said to be the analytical continuation of the former in the field of ordinary experience. This is felt to be a very happy circumstance which bespeaks the unity of physics and encourages the hope that some day a universal theory may be achieved, a hope which at present animates the researches of Einstein. In view of the analytical smoothness with which the two disciplines join it might seem unwise to maintain in one a fundamental attitude which has no place in the other and which would constitute a conceptual break at the passage from one to the other. This argument, too, would suggest an adoption of the objective view. But it should be observed that the points here presented can aim at no more than plausibility, and do not render the opposite view basically untenable.

The opposite view, to be called for brevity the subjective view, is the one which holds that state functions describe our knowledge of physical systems. It is psychologically motivated by the significant observation that the independence of classical states is a fallacy. It springs from the desire of making a clean break with erroneous notions and therefore emphasizes an opposite extreme. Let us first see what can possibly be meant by it. Obviously it can not mean that the state function describes the observer's awareness, his momentary state of mind, and the changes in it which occur in time. It is clear that all this depends on factors far removed from the field of physics. Quantum mechanics certainly does not have the aim of converting physics in to a highly expressionistic type of psychology. Although proponents of this view often employ ambiguous language, what they wish their 


\section{H. Margenau}

states to represent is not actual knowledge but potential knowledge. The state function is to be regarded as a convenient carrier, a symbol for the sum total of all that knowledge which the speculative observer can possibly accumulate, at any instant, with the use of all his resources. It may coincide with that knowledge at certain moments of very acute awareness, that is, when the investigator has made all possible measurements and all calculations pertaining thereto. No one can deny that this view, even in its extreme, has in it a trace of objectivism because of the admission that states refer to potential and not to actual knowledge, and this is a feature which makes it slightly inconvenient to have to defend the subjective position; it demands constant vigilance lest an inadvertant lapse should contradict the initial tenet.

The chief virtue of the standpoint under discussion is that it provides complete safety against positivistic attacks. Its tenant can justly pride himself in being under no obligation to transcendental agencies for transmitting his knowledge, for he deals with nothing but knowledge which he may believe if he wishes to be generated within his mind. He may wonder perhaps why the laws which govern the evolution of potential knowledge operate with almost dynamic regularity, but this is after all no greater a miracle than the existence of rigid laws in an objective world.

The author has not succeeded in bringing to light any further merits of the latter, subjective view. While admitting its possibility on logical grounds, he wishes to record his preference for the former. This preference is partly based on reasons so far presented, partly on considerations of the meaning of probability peculiar to the two attitudes which will now be discussed.

The contrast between objectivity and subjectivity of states has an interesting analogue in the theory of probability, ${ }^{5}$ which, as the reader very likely recalls, can be formulated either as an empirical frequency theory or as a subjective ("a priori”) discipline.

In the former case, probability is defined as the limit, with increasing number of trials, of the relative frequencies of an occurrence. ${ }^{6}$ Probability can be ascertained only empirically, by con-

${ }^{5}$ The philosophical consequences of the quantum mechanical probability formulation have been stated clearly by F. S. C. Northrop, Philosophy of Science, I936, pp. $215 \mathrm{ff}$.

6 For a detailed discussion see Lindsay and Margenau, p. I6I, Wiley and Sons, 1936. 


\section{$35^{\circ}$ Critical Points in Physical Theory}

tinued observations. There are difficulties inherent in this conception, especially mathematical ones connected with the existence of the limit, but it seems that they can be removed. The conception has, however, the great advantage of being applicable to all scientific problems which call for the use of probabilities. Notable also is the circumstance that it permits probabilities to be measured numerically to any desired approximation in accordance with their definition. It is not possible, on this view, to predict probabilitics by a priori considerations peculiar to the probability calculus, although this may in many cases be done tentatively by introducing special physical theories. (Thus the probabilities for tossing heads or tails can be ascertained directly only by making a large number of trials. Yet there is nothing to prevent us from considering the coin as a physical body, then making the assumption of symmetrical mass distribution and supposing a plausible distribution of initial conditions as well as a sufficient variety of them - a basis on which the probabilities may be computed by means of a physical theory.)

But there are cases to which the empirical probability conception can not be applied. They constitute the class of all propositions about single events, such as the statement: it will probably rain tomorrow. Such statements have the peculiarity that no unambiguous measure can be attached to the probability involved, except that, on retrospect, the probability is one if the event has happened, zero if it has not taken place. These cases are covered by what is usually termed the subjective or a priori probability formulation mentioned above. Analysis shows that it always refers to intensity of belief of an individual or to conventional beliefs of groups. Attempts are often made to assign a numerical estimate to such probabilities. In doing so, one of two courses is usually employed. Thinking again of the statement: it will probably rain tomorrow, a measure can be obtained by studying the weather today and then referring to a record of meteorological data for this locality ${ }^{7}$ and finding the relative number of cases in which rain has ensued on a day prior to which the weather agreed with today's. But in this procedure we are

${ }^{7}$ For an analy tic discussion of the meaning of measurement $\mathrm{cf}$. the next section. 
clearly giving up the subjective idea of probability as well as the individuality of the day "tomorrow," which now merges in the statistical aggregate of days characterized by the property that the previous day had weather like the present. Proponents of the subjective view are not always aware of the necessity of a reversion to the frequency concept, typified by the preceding example, which necessity invariably arises when any scientific application is to be made. The other course sometimes adopted for making a priori probabilities statable in numerical terms is to count causes or reasons for or against the occurrence of the event in question. Thus it might be said: 7 causes indicate rain tomorrow, only 2 indicate clear weather, therefore the probability that it will rain is $7 / 9$. But who can determine the relative weights of these causes, or who can say that I have counted the correct number of them (for any given cause can be split up into a number of causes)? In some instances one can say: 7 available theories favor the event, 2 theories oppose it, hence the probability is $7 / 9$. But then, what are we to do with that infinite number of theories, also favoring or opposing the event, which have not yet been invented, or what are the antecedent probabilities of being true for those that have been taken into account? We return therefore to the initial statement regarding subjective probabilities and phrase it thus: Probabilities referring to intensity of belief, or degree of completeness of knowledge regarding a single event, are intrinsically unmeasurable.

Now it is a fact that the student who holds the objective view concerning states is practically forced to couple it with the empirical frequency concept of probabilities, whereas those who incline toward the subjective interpretation of states almost invariably adopt the subjective idea of probability also. The internal compulsion for this alignment is unmistakable. A person willing to assign to a physical system a state independent of his cognition would find it natural also to allow this state numerous modes of empirical manifestation, whereas the knower of a subjective state has room only for greater or lesser certainty of his knowledge. He would say: Now I have made a calculation which makes my knowledge of the electron's momentum certain 


\section{Critical Points in Physical Theory}

to the degree I/2, my knowledge of its position certain to a similar degree; now I have measured the electron's momentum and I know it with complete certainty, while my knowledge of its position is now extremely uncertain, etc. In his opinion this is described in the changes which the state function undergoes.

It must already be apparent that the choice of interpretation of physical axioms is not always a matter of logic, but often of personal preference. So here the correlation of views is not entirely rigid. A mixture is possible in the sense that the last named individual might, while main taining his fundamental stand with regard to the subjective character of states, nevertheless submit his knowledge to experimental tests and thus affix to it an indirect objective measure of certainty. But the view which then results is needlessly complicated and approaches the former in all essential respects. For this reason it does not seem worth recommending. The possible number of combinations of aspects so far presented in this report is already so large that some curtailment becomes imperative.

\section{THE STATISTICAL CHARACTER OF PREDICTIONS}

The considerations in this section are irrelevant to the subjectivist who has adopted the a priori probability idea. We wish here to deal with the question: granted that the probability predictions which arise in quantum mechanics refer to a statistical aggregate in the spirit of the frequency theory, what type of aggregate is meant? It is clear that it must be an aggregate of physical observations, indeed in the ideal limit an infinite number of observations, or measurements. ${ }^{7}$ Surveying the field, we find after little search that three types of collections of observations present themselves:

I. Numerous simultaneous observations of a given quantity when the system is in a given state;

2. Numerous observations, or a single collective observation, on a physical assemblage of many similar systems in the same state;

3. Numerous repeated observations on the same system, the state in question being reprepared before each observation. 


\section{H. Margenau}

The first of these is only a speculative possibility, for there are many types of observation which can be carried out but once and permit no simultaneity with any other measurements even of the same type. Hence our choice is reduced to possibilities (2) and (3).

The contrast between these is best illustrated by an example. Let the state function for the system "electron" be such as to imply: the probability of observing its position in a certain specified volume is I/IO. According to (2) this means that if I take Iooo electrons all in the state under consideration (we suppose at present that I know how to prepare this state) Ioo of these electrons will lie in the specified volume. According to (3) it means that if an electron is in the state in question, I may find it in the specified volume or not, but if I prepare the state I000 times, I shall find it there approximately Ioo times. In (2) the probability aggregate is a collection of observations on a physical assemblage, in (3) it is a collection of observations on an ideal ensemble consisting of an infinite repetition of a set of physical operations. The same fundamental distinction occurs at one other place in theoretical physics, namely in the approaches to thermodynamics by Darwin and Fowler on one hand, and by Gib.bs on the other.

The decision here to be made clearly involves the question whether or not quantum mechanical reasoning is at all applicable so single systems. If it is not, (3) must be abandoned. Otherwise both might be acceptable, but (2) might be a consequence of (3). Now it is certainly true that in most physical observations dealing with atomic phenomena indirect means are employed, and the number of systems affected is very great indeed. The energy of an atom can be measured for instance by allowing it to radiate and then analyzing the radiation. But when the spectrum is to be photographed the radiation from a single atom is far too weak to register itself. Hence a great number of atoms must be admitted. It is found that the spectrum always contains many different frequencies. Does each atom emit all these frequencies, or does each emit a single frequency, the different ones coming from different atoms? Experiment does not provide the 


\section{Critical Points in Physical Theory}

answer directly, but we shall find it by considering another situation.

Quantum mechanics predict for the coordinate (probability) distribution of an electron which has passed through a narrow slit, a diffraction pattern, i.e. well defined maxima and minima in a plane behind the slit. If, in order to test this result, a beam containing many electrons is used, the pattern can be observed by means of appropriate devices, like ionization chambers. By this experiment the question whether a large number of electrons is necessary to produce the probability pattern can not be decided. It is feasible, however, to make the electron beam very weak, so weak in fact that the electrons constituting it are widely separated in space and cannot be supposed to interact with one another. Under that condition the predicted distribution is also observed, but now as a frequency distribution among the positions of capture of the individual electrons, the captures being altogether independent events. The conclusion is therefore inescapable that the statistical feature of this process is attached to the individual electron, and that, even if the same state were prepared again for the same electron, it, too, would show a dispersion of positions. And this means that quantum theory deals with an aggregate characterized by (3), not necessarily by (2).

Several other experiments could be adduced to confirm the proposition that the theory is applicable to single systems. We shall content ourselves with one further point. It is well known, and demonstrable in many ways, that a light signal which is emitted in a very short time cannot be monochromatic but must have a wide spread in frequencies. Modern theory bids us consider the light flash as a photon, a single photon if it is sufficiently weak, and its frequencies as being proportional to energies. If now that well-known fact is to be preserved, we must, in quantum mechanics, associate many different energies with a single photon; we must assume that a photon in a state defined by its sudden generation must exhibit sometimes one, sometimes another energy to the observer.

We can now say what happens, presumably, when many atoms emit a spectrum. One atom would generate the entire spectrum 
if it were given sufficient time to register its radiation on a photographic plate. Of course a single photon would fall on only one spot of the plate, but another photon would hit another spot, and so on, until finally the whole plate is blackened in accordance with predicted probabilities. The supposition is that the atom is returned to the same state before each act of emission, and this may be done, for example, by exposing it constantly to another suitable source of radiation.

It is clear, then, that the statistical aggregate in question can not be defined by (2) at the exclusion of (3). The latter is certainly a correct interpretation. On the other hand, no one will deny that possibility (2) is somehow more graphic and more plausible. The reason for this is not far to seek. It is simply because the older classical statistical theories have familiarized physicists as well as philosophers with the notion of assemblages (e.g. gases) which exhibit probability features only by virtue of possessing hosts of constituents. There every constituent obeys strict dynamical laws, statistics arise from our ignorance of certain parameters, such as the initial positions and momenta of all the molecules, and the argument goes that if these parameters were known the use of probabilities would be unnecessary. In quantum mechanics, as we have shown, the situation is altogether different; no search for hidden parameters in the ordinary dynami cal sense can ever undo the probability thesis-unless the theory itself is abandoned or essentially modified. This follows simply from the fact that in quantum theory the single system, which is the carrier of the statistical aggregate, can by definition not be further decomposed into entities with unknown properties.

If (3) defines the aggregate to which the theoretical probabilities refer, what about the aggregate (2)? A moment's reflection will show, we believe, that it is a special case of the former, provided we are willing to make a slight but very significant change. In (3) we spoke of repeated observations on the same system. There are many reasons for believing that one ultimate system, like an electron or a proton or a neutron, is physically indistinguishable from another system of the same kind. Therefore it seems permissible to loosen the strictness of the word same into a sort of 


\section{Critical Points in Physical Theory}

of promiscuous identity, designating by the same system merely a system of the same kind. The collective measurement on a physical assemblage of similar systems, contemplated in (2), may then be regarded as a sequence of almost simultaneous measurements on the "same" system in the new sense of (3). Item (2) thus becomes a special case of item (3). But item (3) expresses the basic issue.

\section{THE THEORY OF MEASUREMENTS}

A. Meaning of measurement. The usual way to theorize about measurements is to select, for no obvious reason at all, a specific type of experiment, analyze it very fully, and then generalize the results without inquiring very much whether these results fit the multitude of other measurements that might be considered. The experiments chosen are mostly imaginary ones, a feature which of course detracts in no way from the value of the example as long as the imaginary procedures are permitted by known physical laws. Heisenberg's experiment for determining the position or the momentum of a particle by means of a $\gamma$-ray microscope, and Bohr's slit experiment have perhaps borne the brunt of this procedure. Not as common but yet quite popular, particularly among mathematicians, is a mode of inquiry which starts with quantum mechanical theory, considers what ideally a measurement ought to be, and then manages to find one which fits the prescription. In studying J. von Neumann's excellent book ${ }^{8}$ on the foundations of quantum mechanics we have had the impression that this method was being employed. What makes the method seemingly successful is the wealth of existing types of measurement; it insures that any prescription can be filled.

Let us here try to avoid these procedures, let us begin by analyzing empirically, and on a sufficiently broad basis, what the physicist means when he says he makes a measurement. The breadth of the issue is at first bewildering, but it seems that the following definition covers it completely: a measurement is any physical operation by means of which the numerical value of $a$

${ }^{8}$ von Neumann, Mathematische Grundlagen der Quantum mechanik. 


\section{H. Margenau}

physical quantity can be determined. This wording is not meant to suggest that physical quantities are always or sometimes "real," or that they possess at any instant unique numerical values. We know that they do not, and we refuse as physicists to join the irrelevant altercations about reality. What matters is that there are generally accepted physical operations whose outcome is associated with a certain number, and this number is commonly referred to as the value of some quantity. All dangers are avoided if the reader thinks of "value of a quantity" as a figure of speech. The definition shows that a measurement is different from a physical operation and an observation, measurement being more specific than either of the last terms, while an observation differs from an operation in being a purely passive act of perception. A measurement usually consists of an operation plus an observation, but this need not be the case.

The present purpose is best served by leaving aside altogether measurements on systems of the immediately perceptible domain, i.e., on large scale bodies, and to concentrate our attention at once upon the more problematical part of our question, the measurement on atomic systems, atomic in the original sense of the word. Our main endeavor is to show how rich is the field of possible procedures.

The simplest quantity to be measured on any system is perhaps its position. Among the possible ways of effecting a position measurement one seems to be most commonly employed: the method of absorption. If the system is an electron, Faraday cages or similar devices might be stationed at various places, and the place of the one which registers is the place where the electron has impinged. If the system is a photon, a photographic plate is used, and the place of the photon's impact is indicated by certain chemical changes. (We are not concerned at present with limits of accuracy of measurements.) Next let us turn to the momentum of a single system, say an electron or a photon. Perhaps the simplest way of measuring it is to allow the particle to be diffracted by a suitable grating and then to determine its impact on a plate, i.e. its position in the manner just discussed. The energy can of course be found in a similar fashion. These 


\section{Critical Points in Physical Theory}

experiments are more complicated than the foregoing ones inasmuch as they require the addition of another stage or operation to the position measurement, and this seems to be a typical feature of most measurements. It is to be observed that the phenomenon of diffraction alone is not a momentum measurement in accordance with our definition, for unless it is coupled with the absorption of the photon on a plate no number can be ascertained. We deem this fact of some importance despite the carelessness with which the matter is often treated, and we shall have occasion to return to it in another connection.

It would be folly to suppose that all physicists will consider the simple measurements here chosen for discussion as more basic or more representative than others. The point to be made is that they are bona fide measurements of very common occurrence. They present an interesting feature: after the measurement the system can no longer be said to be present as an identity. This is particularly true in the case of the photon which after absorption ceases to function as a physical system altogether, while the electron may in a sense be said to be "still there," but has become one of a large assemblage of indistinguishable electrons inside a metal. We note, then, that some measurements annul a system, others render them of no physical interest with respect to the measurement in question. Further examples of measurements which annul the system would be the determination of the place of a neutron (by nuclear capture) and certainly that of a neutrino, if this should prove to be a useful construct. Cases of this type arise of necessity when systems are introduced which can be created or destroyed.- Throughout this discussion the language of classical physics has been used. In terms of it we might state the matter thus: the classical fate of systems during measurement is not describable in a uniform way; an attempt to describe it completely would introduce irrelevancies. We shall see that quantum mechanics, with its altered definition of states, relieves us of all embarrassment in this connection.

The number of legitimate measurements in physics is theoretically unlimited, as is easily seen from the fact that every new discovery, every new verified theory provides new opportunities for 


\section{H. Margenau}

measuring old quantities. For instance, a Wilson cloud track would not have been a means for measuring the energy of an $\alpha$-particle before Wilson's discovery of the condensation of water vapor on gaseous ions. Nor would Heisenberg's $\gamma$-ray microscope have been a suitable device for measuring an electron's position before the Compton effect was known. What is now termed a measurement would then have been merely an experiment. In view of the derivability of new kinds of measurement from special discoveries, the temptation is strong to standardize the theory of measurement by picking out a certain basic group, perhaps to be called the primary group, on which may then be built an unlimited system of secondary measurements. Sucn an analysis would be extremely desirable for logical as well as linguistic reasons, but will not be attempted here because the selection of primary measurements would be an arbitrary undertaking.

Besides measurements, there is another very important class of physical operations: the preparation of states. We are not at this moment interested in such operations, but we do not wish measurements to be confused with them. For example, when electrons are made to pass in to a magnetic field, a new state with respect to electron spin has been produced, but the spin has not been measured.

A word might be said about the compatibility of measurements. The literature abounds with statements to the effect that simultaneous measurements on complementary quantities (in Bohr's sense) are incompatible. If this is interpreted as meaning that they cannot be made the statement is either without point or it is wrong. For in that class of measurements which annul a system no simultaneous ones are possible at all and the proposition seems void. On the other hand there is nothing to prevent an inquisitive observer from illuminating an electron simultaneously with hard and soft quanta, and if, according to the orthodox view, the hard quantum on being scattered tells the electron's position and the soft one its momentum (Doppler effect), two "incompatible" measurements have been performed. We know that the results are not to be trusted and that, on repetition, the measured values would scatter greatly, but the example does 


\section{Critical Points in Physical Theory}

constitute an exception to the general claim of incompatibility for (simultaneous) complementary measurements. What the phrase should mean is that such measurements have a predictable spread. ${ }^{9}$

There appears to be a tendency to regard quantities of a physical system which can be measured collectively without spread in the previously defined statistical sense, as real quantities, others being unreal. This is the most peculiar use of a metaphysical term, a term which would be objectionable in physics even if it were used more correctly. It springs very clearly from a failure to abandon classical notions where they should be dismissed. In classical mechanics, when a definite ultimate state is given, an elementary quantity which refuses to yield the same value every time it is measured is a self-contradiction, is unimaginable. In quantum mechanics such things do occur, and the success of the formalism has forced many classically-minded physicists to accept it. But their displeasure still prevents them from acquiescing completely, and they feel better when they are able to give things a private little knock by calling them unreal.

B. Measurement and Uncertainty. The mathematical meaning of the "uncertainty principle" is perfectly clear and requires no comment. To wit: when any state function is given, the uncertainty - a term which has no precise accepted significance but can be defined suitably-may be computed numerically. Ambiguity prevails only as to the interpretation of the numbers which so result.

Here again account has to be taken of the two possible meanings of probability. On the subjective view, the uncertainty affects a single measurement, but it can in that case be nothing more than a qualitative subjective estimate, clad in numerical form but incapable of verification, of the assurance with which certain results may be expected. Every other interpretation would at once revert to the position which envisages the single measurement as the constituent of a statistical aggregate, and this would

\footnotetext{
9 In making such predictions the exact meaning of the word "simultaneous" must be known. The results of the measurements depend on the order in which they are performed because the operators in this case are not permutable.
} 
necessarily be the one discussed in section III, (3). The usual statement according to which an uncertainty represents an error is meaningless on this view, for a single measurement contains no error, or, more explicitly, no verifiable error. The present position, if maintained consistently, is by no means illogical; but it fails to utilize a wealth of formal structure potentially present in quantum theory.

If, on the other hand, the probability concept is accepted in its empirical sense, uncertainty can truly be interpreted as an error. The uncertainty principle (for the sake of definiteness let us think of it in the form $\left.\Delta p \cdot \Delta q \geqq \frac{h}{4 \pi}\right)$ then relates the spread, $\Delta \mathrm{p}$, occurring in numerous momen tum measurements on similarly prepared systems, to the spread $\Delta \mathrm{q}$ in corresponding position measurements. On this view the principle becomes far more explicit, it exposes itself in a simple way to empirical verification, which, by the way, lends it the strongest support. The inequality sign appearing in the usual form of the relation, or the sign $\sim$ which is also frequently used, does not imply that $\Delta p$ or $\Delta \mathrm{q}$ are not known with precision; indeed in any given case both $\Delta \mathrm{p}$ and $\Delta \mathrm{q}$ can be computed exactly from a knowledge of the state function; and the computed values are independent of the kind of measurements to be used for their determination.

Most physicists consider it very important to secure a plausible classical explanation of the uncertainty relations, which fundamentally are nothing more than a by-product of the quantum mechanical description of nature. There is indeed no harm in seeking such an explanation, but in doing so one should be aware that the procedure involves basically a fusion (and often a confusion) of incongruous physical concepts: the classical and the quantum mechanical ideas of a state. In this connection it is well to remember that the uncertainty relations were implicitly used before they were discovered. The common claim is that they express the uncontrollable error introduced into physical description by the interaction between system and measuring devices in the act of measurement. The physical facts underlying this assertion are undoubtedly correct, but the statement is 


\section{Critical Points in Physical Theory}

unfortunate in the light of a more meticulous analysis of the meaning of measurement. We have seen that the theoretical uncertainties are completely independent of the choice of measuring devices, whereas measurements affect a system in a perplexing variety of ways. Uncertainties are in a very definite sense inherent in a state even before measurements have been made. The correct statement therefore is that the uncertainty relations reflect the interaction between system and apparatus when the state is being prepared, not when it is measured. A closer inspection of the arguments presented in the literature for the former loose assertion reveals that its defenders perhaps unwittingly mean the latter. Logically, however, there is a difference, for the former position inevitably leads to a theory which counts the disturbing interactions twice, once in the preparation of the state, once in its measurement. What quantum mechanics actually does is to stop all inquiry at the act of measurement.

We feel that the matter is really quite simple and that most argumentation on this issue has resulted from a failure to define concepts clearly. The most common artificial complication arises in this way. Numerous measurements (not all) have of course the property of preparing a new state, a fact which is of no in terest if $I$ agree to regard the act in question as a measurement. If, however, by a mental lapse I forget to efface all in terest after this event and continue to question what happens, then I have contradicted the original premise according to which I was making a measurement and am regarding the act as an operation preparing a state. The practically-minded physicist will probably regard these distinctions with mild amusement-but we are, after all, dealing with the finer points of modern theory.

The problem of causality, which naturally raises its head at this place, has been dealt with previously ${ }^{10}$ from this point of view. We have no cause to modify the conclusions then presented.

C. Does a measurement produce an eigenstate? The reader will recall that an eigenstate of an observable quantity is a state for

${ }^{10} \mathrm{H}$. Margenau, Journal of Phil. of Science, 1, I33 (I934). 
which the measurement of a single value of that observable quantity can be predicted with certainty. In other words, the probability distribution with respect to that observable, which can be computed from the state function, degenerates into zeros for all values but one. Now orthodox theory claims ${ }^{11}$ that a measurement, performed upon a system in an arbitrary state, immediately and suddenly converts this state into an eigenstate with respect to the quantity measured. This change is held to be indescribable by any theory and essentially unpredictable. Troublesome questions as to the length of time which must be allowed for this sudden change, whether it takes place discontinuously in zero time or whether it is somehow related to the kind of measurement enacted, have already arisen but are left unanswered because quantum theory is felt to be not sufficiently complete to deal with them. This doctrine recognizes two possible ways in which a state function may be altered; first, continuously in accordance with the Schrödinger equation, the change being uniquely predictable; second, in an unpredictable way when a measurement is performed. The eigenstate thus generated is of course the one corresponding to that particular value of the observable which the measurement has yielded. On this theory of unpredictable transitions is often based the assertion that quantum mechanical description is an essentially acausal one.

Let us view this matter in the light of the analysis conducted in this paper. It is then perfectly clear that our attitude here is decided by the fundamental choice made with respect to the interpretation of states. If we adopt the subjective view, according to which a state function is a symbol merely for knowledge, and couple it with the subjective view of probabilities-and only if this choice is made- the theory of acausal jumps is indeed correct, for a measurement converts uncertain knowledge in to certainty. It is interesting to observe that this change occurs as the result of a single measurement. Therefore the probability interpretation attending the whole process must be the one which endows the probability of single events with meaning, and it entrains

" Cf. for instance von Neumann, Mathematische Grundlagen der Quantenmechanik, Schrödinger, Naturwissenschaften 23, 823 (1935). 


\section{Critical Points in Physical Theory}

those consequences, such as lack of measurability, which we have discussed in section II. We have already indicated that in our opinion such consequences are unfortunate, but if they are accepted, the orthodox view is entirely logical.

On the other hand, let us see what happens if we regard states in the objective sense, and measurements as revealing states in a statistical manner, the statistical aggregate being the one exposed in section III. The theory of acausal jumps is then decidedly to be rejected. For in the first place a single measurement allows no inference as to the state at all. In the second place, we have seen that measurements, if described in classical language, affect systems in such a large variety of ways, sometimes even annulling them completely, that they cannot be said to produce an eigenstate of an observable in a uniform manner. It is the great merit of quantum mechanics that it permits a description of experience without forcing us to take account of the sometimes disastrous results of a measurement. Classical physics required the investigator to be ever on the guard for disturbances occurring when he made his measurements. He maintained his vigilance in the mistaken belief that such disturbances could be controlled, but had to capitulate when this belief was shaken. The new theory permits us to forget these difficulties completely by incorporating a suitable compensation mechanism in its fundamental structure: the method of describing measurements statistically. On the objective view, the assertion that a measurement produces an eigenstate is precisely as meaningful as the contention that, after cracking and eating a nut, I still have a nut, but in its cracked and eaten state. In an earlier paper ${ }^{12}$ the present attitude was briefly formulated, but sufficient reasons for its acceptance were not given.

Perhaps it is well to remark in some detail on a few arguments which often serve as a basis for maintaining the objective view of states, together with the theory of acausal transitions. One, illustrated with the use of an example, runs as follows: I wish to measure the state of polarization of a photon, about which I know

${ }^{12}$ H. Margenau, Phys. Rev., 49, 240 (1936). 
nothing initially. For this purpose I send it through a Nicoll prism, an analyzer. If it passes through I have made the measurement, for I then know its plane of polarization to be the plane in which the Nicoll transmits. But if I station another analyzer immediately behind the first, with its axis parallel to that of the first, I am absolutely certain that it will go through the second Nicoll also. Hence the first measurement has produced an eigenstate with respect to the photon's state of polarization. The error lies in forgetting that $I$ have not made a measurement until I have ascertained, by catching the photon on a plate, that it has gone through the Nicoll. The error is simple but essential, it springs from a hazy conception of what is meant by a measurement, and this, incidentally, is the cause of many similar difficulties.

Occasionally one finds the position, already shown to be untenable in section III, that quan tum mechanics deals with actual assemblages of similar systems, like a swarm of electrons, in some state. If a measurement is then performed, a sub-assemblage is selected from the former group of systems, this sub-assemblage comprising all those individuals, which have the measured property. It is this new, smaller assemblage to which the state function after the measurement refers, and this is clearly in an eigenstate. So goes the contention. Three comments should be made with regard to it: (I) quantum mechanics is capable of dealing with single systems and is not limited to assemblages; (2) very few measurements have the property of selecting sub-assemblages, on which the above argument rests; (3) a state function in quantum mechanics refers always to the same number of individual systems, i.e., to the same assemblage. This argument is even weaker than the foregoing one and is indeed also less common.

The real backbone of the theory of acausal jumps is probably to be found, however, in a peculiar mathematical attitude which gives way to a tendency of securing physical counterparts to mathematical notions whether these counterparts fit into the physical scheme or not. To wit: the mathematical theory of quantum mechanics is full of operators, that is, to every physical observable there corresponds an operator. But very few of the 


\section{Critical Points in Physical Theory}

available mathematical operators are accommodated in this way, are ennobled by being physically established. There is in particular a very beautiful and general class of operator, the projection operator (terminology comes from the theory of Hilbert space) which converts a function into what amounts to an eigenstate-function. This operator fairly cries for physical accommodation. What is more suggestive than to associate it with measurement in general? This correlation, once tentatively made, finds support in the fact that there are, as we have seen, some measurements which, if they are wrongly interpreted as operations producing states, do produce known eigenstates. Hence the theory which we are critizing. We have here encountered another example where a theoretical suggestion, supported by a few empirical instances, is blindly generalized into an allembracing law.

The view that a state function jumps unpredictably during measurement may lead to monstrous consequences of which only one will be considered. Unless a measurement is cleanly separated from other physical operations - and this separation, as we have seen, involves a rejection of the jump theory-one is induced to reason as follows: a measurement is, after all, the result of an interaction between the system and some physical apparatus. If, then, this same physical process is allowed to take place but without the understanding that it shall constitute a measurement, the state function describing it will develop continuously in accordance with the Schrödinger equation; if the process is regarded as a measurement it will change abruptly and indeterminately. Where is the line to be drawn? One might for instance be looking at a recording device and, while day-dreaming, fail to take conscious cognizance of the registration. All physical processes, as the term is ordinarily interpreted, are the same as if he were taking conscious notice of the result, but in one case the state function develops continuously, in the other it changes abruptly. This conclusion is clearly not interpretable unless we are willing to say that the state function means knowledge. If the absurdity is justified, as is sometimes done, by the observation that processes in the human brain do matter and that 


\section{H. Margenau}

the ego is to be introduced into the scheme somewhere, the only significant reply is, we believe, that quantum mechanics does not as yet pretend to be a psychological theory. As such it would have to show a little more competence in the purely psychological realm. Again the solution of the apparent dilemma is perfectly simple: There are no acausal transitions of states; if I consider the experimental procedure as a measurement I should refrain from talking about it as though it were not. I must then stop my description of the state after taking cognizance of the measured value. On the other hand, if the procedure is not a measurement I can certainly (at least in principle, actually the description it very difficult and usually impossible) continue to trace the state function as long as I desire in definite fashion; the registration which has occurred incidentally in this procedure does not, as we now know, determine the state function completely, nor could it in general have been predicted from a complete knowledge of the state function.

Of some interest in the present context is a mathematical process known as the "method of reduction of the wave packet." Its exact nature will not be discussed in great detail..13 Its usual application to systems containing two elementary particles makes use of the "jump theory" in this way. The state function of the pair is, by reasion of a past interaction, such that it can not be written as a product of two functions each containing the coordinates of but one particle, but appears as a sum of such products. When a measurement on one particle is made, the state function corresponding to that particle is known: it represents the eigenstate belonging to the measured value. But this function appears in the original sum as multiplied by a coefficient which contains only the coordinates of the other particle, and which can therefore be regarded as its state function after the measurement. Thus, it is contended, a measurement causes the originally mixed function to reduce itself suddenly to a simple form, thus "resolving the wave packet."

On the present view, no such event happens; it can only be

${ }^{13}$ See the appendix, II, for those parts which are here of interest. 


\section{Critical Points in Physical Theory}

shown-and this is done in the appendix ${ }^{13}$ - that there exists a peculiar correlation between the probabilities for the various results of measurements. It is this: When suitable measurements on the two particles are made, the probability for the occurrence of a pair of values is zero unless the pair is the one which corresponds to the two eigenfunctions of the reduced package. The physical consequences are the same as on the customary view, but the result appears now in less metaphysical language. The method of the reduction of the wave package remains in good practical standing, only its interpretation requires greater care.

It is hoped that the analysis, which is herewith concluded, may contribute toward a more precise understanding, a deeper appreciation of the internal logical consistency, and a clearer linguistic formulation, of recent physical theories.

\section{APPENDIX}

I. The state function $\phi$ of the electron, if it were known, could be expanded in terms of the eigenfunctions $\psi_{\lambda}$, of any other operator A, as follows:

$$
\phi=\sum_{\lambda} a_{\lambda} \psi_{\lambda}
$$

The $a_{\lambda}$ are complex numbers, to be interpreted as follows: the square of the absolute value of any particular one, say $a_{k}$, is equal to the probability that a measurement of $A$ yields the $k^{\text {th }}$ eigenvalue $A_{k}$.

Suppose now that $\phi$ is not known, but that all $\left|a_{\lambda}\right|^{2}$ are known from experiments on $A$. The value of every $a_{\lambda}$ is then uncertain with respect to a factor of absolute value I. If we attempt to construct eq. (I) we can at best only get

$$
\phi^{\prime}=\sum_{\lambda} a_{\lambda} e^{i \delta_{\lambda}} \psi_{\lambda}
$$

with all the $\delta_{\lambda}$ 's unknown, except that they must be real.

The important point is that ( $\mathrm{I}$ ) predicts a different probability distribution for another observable with operator $B$, than does (2). For if $\chi_{\mu}$ is the sequence of eigenfunctions belonging to $B$, then the probability of measuring the eigenvalue $B_{k}$ when the electron is in the state $\phi$ will be

$$
\left|\int \phi \chi_{\mathbf{k}}^{*} \mathrm{~d} \tau\right|^{2}=\left|\sum_{\lambda} \mathrm{a}_{\lambda} \int \psi_{\lambda} \chi_{\mathrm{k}}^{*} \mathrm{~d} \tau\right|^{2}
$$




\section{H. Margenau}

But when the system is in the state $\phi^{\prime}$ which is experimentally indistinguishable from $\phi$, this probability is

$$
\left|\int \phi^{\prime} \chi_{k}^{*} \mathrm{~d} \tau\right|^{2}=\left|\sum_{\lambda} a_{\lambda} e^{i \delta_{\lambda}} \int \psi_{\lambda} \chi_{k}^{*} \mathrm{~d} \tau\right|^{2}
$$

(3) and (4) are equal only under two conditions:

(a) when all $\delta_{\lambda}$ are equal

(b) when the sum in (4) reduces to a single term.

Case (a) is an accident which is not under our control. Case (b) can arise in one of two ways: Either the integral $\mathcal{S} \psi_{\lambda} \chi_{k}^{*} \mathrm{~d} \tau$ is zero for all but one value of $\lambda$. Then $\chi_{k}$ is essentially the same as one of the $\psi$ 's, and we are measuring the observable $\mathrm{A}$ over again. It is small wonder that we can predict what we already know! Or every $a_{\lambda}$ is zero except one. This is the situation discussed in the text. It means that the first operator A was one for which the measurements did not spread.

II. Let the state function after the interaction be

$$
\psi\left(\mathrm{x}_{1} \mathrm{x}_{2}\right)=\sum_{\lambda} \mathrm{v}_{\lambda}\left(\mathrm{x}_{2}\right) \mathrm{u}_{\lambda}\left(\mathrm{x}_{1}\right)
$$

Here $x_{1}$ and $x_{2}$ denote the coordinates of the two particles, respectively; $u_{\lambda}$ is an eigenfunction belonging to the eigenvalue $A_{\lambda}$ of the operator $A$; $v_{\lambda}$ is (a constant times) an eigenfunction belonging to the eigenvalue $B_{\lambda}$ of the operator B. A "biorthogonal" development like (5) is sometimes possible, ${ }^{14}$ it has been made the basis of certain metaphysical conclusions by Einstein, Rosen and Podolski ${ }^{15}$ which we shall not discuss further. ${ }^{12}$ We are here not interested in other cases. The probability that a simultaneous measurement of $A$ on particle (I) and of $B$ on particle (2) shall yield the pair of values $A_{i}$ and $B_{j}$ is, by the principles of quantum mechanics,

$$
\begin{aligned}
& \left|\iint \psi\left(\mathrm{x}_{1} \mathrm{x}_{2}\right) \mathrm{u}_{\mathrm{i}}^{*}\left(\mathrm{x}_{1}\right) \mathrm{v}_{\mathrm{j}}^{*}\left(\mathrm{x}_{2}\right) \mathrm{d} \mathrm{x}_{1} \mathrm{~d} \mathrm{x}_{2}\right|^{2} \\
= & \left|\sum_{\lambda} \int \mathrm{v}_{\lambda}\left(\mathrm{x}_{2}\right) \mathrm{v}_{\mathrm{j}}^{*}\left(\mathrm{x}_{2}\right) \mathrm{d} \mathrm{x}_{2} \int \mathrm{u}_{\lambda}\left(\mathrm{x}_{1}\right) \mathrm{u}_{\mathrm{i}}^{*}\left(\mathrm{x}_{1}\right) \mathrm{d} \mathrm{x}_{1}\right|^{2} \\
= & \left|\int \mathrm{v}_{\mathrm{i}}\left(\mathrm{x}_{2}\right) \mathrm{v}_{\mathrm{j}}^{*}\left(\mathrm{x}_{2}\right) \mathrm{d} \mathrm{x}_{2}\right|^{2}=\mathrm{c} \delta_{\mathrm{i} j},
\end{aligned}
$$

${ }^{14}$ Cf. Schrödinger, Proc. Camb. Phil. Soc., 31, 555, (1935).

${ }^{15}$ Einstein, Rosen, and Podolski, Phys. Rev., 47, 777 (1935). 


\section{Critical Points in Physical Theory}

where $\mathrm{c}$ is a constant and $\delta_{\mathrm{ij}}$ the Kronecker $\delta$-function. We are supposing for simplicity that all states occurring here are non-degenerate. It is thus seen that the probability of measuring $A_{i}$ and $B_{j}$ is zero unless $i$ and $j$ characterize two functions paired in (5). This is all that matters in any application of the method of resolving the wave packet. The conclusion that the state function makes an abrupt transition is quite irrelevant and avoidable.

\section{REFERENCES}
A. Einstein, B. Podolski, and N. Rosen, Phys. Rev., 47, 777 (1935).
E. C. Kemble, Phys. Rev., 47, 973 (1935).
A. E. Ruark, Phys. Rev., 48, 466 (1935).
N. Bohr, Phys. Rev., 48, 696 (1935).
E. Schrödinger, Naturwissenschaften, 23, 807 (1935).
E. Schrödinger, Naturwissenschaften, 23, 823 (1935).
E. Schrödinger, Naturwissenschaften, 23, 844 (I 935 ).
E. Schrödinger, Proc. Camb. Phil. Soc., 31, 555 (1935).
H. Margenau, Phys. Rev., 49, 240 (1936).
W. H. Furry, Phys. Rev., 49, 393 (1936).

Yale University,
New Haven, Conn.

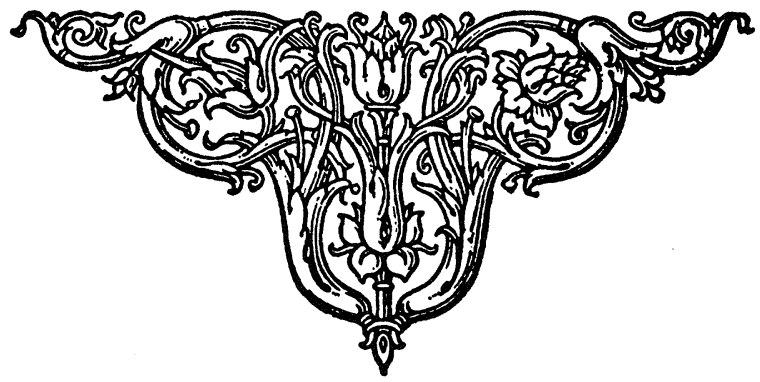

